\title{
El uso del patrimonio documental para Educación Primaria y Secundaria: talleres didácticos en archivos históricos
}

The use of documentary heritage for Primary and Secondary Education: educational workshops in historical archives

José Ignacio Ortega Cervigón (*)

\section{Resumen}

El objetivo es encial del estudio es promover la utilización de documentos y fuentes primarias custodiados por l os ar chivos como elementos válidos par a formular hipótesis s obre la construcción del conocimiento histórico. La investigación muestra la necesidad de aunar el uso de las fuentes y la aportación didáctica de los archivos para valorar el patrimonio documental en la didáctica de la historia. Los principales resultados sintetizan la realización de distintos talleres didácticos adaptados a distintos niveles académicos en archivos históricos, c on el des arrollo de ac tividades y su evaluación. Las conclusiones confirman la obtención de conocimientos sobre la vida cotidiana de los grupos sociales y el aprendizaje por los alumnos de conceptos esenciales del tiempo histórico como la multicausalidad, el cambio y la continuidad.

Palabras Clave: didáctica de $\mathrm{I}$ a $\mathrm{H}$ istoria, $\mathrm{f}$ uentes primarias, ar chivos, $\mathrm{t}$ aller didáctico, $\mathrm{p}$ atrimonio documental, Educación Primaria, Educación Secundaria

\begin{abstract}
The es sential objective of the study is to promote the use of doc uments and primary sources kept by the archives as $v$ alid el ements to formulate $h$ ypotheses about the $c$ onstruction of hi storical $k$ nowledge. The investigation shows the need to combine the use of sources and the didactic contribution of archives to value the $\mathrm{d}$ ocumentary $\mathrm{h}$ eritage in $\mathrm{H}$ istory instruction. The $\mathrm{m}$ ain $\mathrm{r}$ esults $\mathrm{s}$ ynthesize the $\mathrm{r}$ ealization of $\mathrm{d}$ ifferent educational workshops adapted to different academic levels in historical archives, with the development of activities a nd their e valuation. The conclusions confirm the obtaining of knowledge about the da ily life of social groups and the learning process of students about some basic historical time categories such as multicausality, change and the continuity.
\end{abstract}

Key words: History teaching, primary sources, ar chives, e ducational workshop, d ocumentary heritage, Primary Education, Secondary Education.

\section{Introducción}

Uno de I os objetivos primordiales de I a di dáctica de I as Ciencias Sociales es fomentar el pensamiento social y lograr la comprensión del tiempo histórico a partir del análisis de las fuentes primarias. A ún en el si glo XXI de las autopistas de I a i nformación, el desco nocimiento de I os archivos históricos como g uardianes de un pa trimonio docu mental ingente y su s posibilidades

\footnotetext{
. Universidad Complutense de Madrid, joseiort@ucm.es, ORCID 0000-0002-2520-7329
}

Ortega Cervigón, J. I., (2021). El uso del patrimonio documental para Educación Primaria y Secundaria: talleres didácticos en archivos históricos. Clio. History and History Teaching, 47, 295-314. https://doi.org/10.26754/ojs clio/clio.2021475503 Recibido: 8/6/2021. Aceptado: 23/11/2021. 
didácticas es aún una barrera por explorar y del que extraer experiencias didácticas significativas. Los documentos custodiados por los diferentes archivos muestran toda la riqueza temática de los contenidos y procedimientos curriculares y so $\mathrm{n}$ un punt o de par tido i nmejorable par a formular hipótesis sobre la construcción del conocimiento histórico.

Para ello se nos antoja indispensable una visita académica del área de las Ciencias Sociales, bien en el ci clo final de $E$ ducación $P$ rimaria, bi en durante I os cursos de I a $E$ ducación $S$ ecundaria Obligatoria, a un archivo histórico en el que los alumnos y las alumnas puedan conocer de primera mano sus funciones, qué tipo de documentos albergan, el método de investigación histórica y la adquisición de valores de respeto del patrimonio cultural y documental.

Describiremos cómo realizan talleres didácticos adaptados a distintos niveles académicos los profesionales del $\mathrm{A}$ rchivo $\mathrm{H}$ istórico $\mathrm{P}$ rovincial de Cuenca y anal izaremos parte del material elaborado por las propias instituciones archivísticas para lograr los objetivos marcados con vistas a obtener un aprendizaje óptimo de la visita didáctica. A través de ejemplos concretos -mapas, planos, docu mentos históricos, cu adros genealógicos, et c.-, se puede $\mathrm{n}$ desa rrollar y co nstruir conocimientos sólidos sobre la vida cotidiana de las sociedades históricas.

También es una a ctividad adecu ada para anal izar al gunos temas instalados en el i maginario colectivo tanto del profesorado como de los y las discentes y desmontarse o al menos matizarse convenientemente desde el análisis de las fuentes archivísticas. La utilización de fuentes primarias facilita al alumno conocer las variables sociales, el concepto de multicausalidad y el relativismo del conocimiento hi stórico. El ar chivo es un y acimiento di dáctico en el que plasmar la metodología empática en el que descubrir las pervivencias o las transformaciones del pasado. El conocimiento del $\mathrm{m}$ étodo de trabajo del hi storiador y de la hi storiadora i ntroduce I a adquisición de I a competencia científica y facilita el pensamiento necesariamente crítico que es fundamental para lograr profundizar en los contenidos conceptuales y asimilar procesos y habilidades en la obtención de conocimientos históricos.

\section{Marco teórico}

\subsection{La investigación en Didáctica de las Ciencias Sociales y el uso de las fuentes primarias} en Historia

La I ínea de i nvestigación en di dáctica del pat rimonio se ha er igido en una de I as cinco $\mathrm{m}$ ás importantes dentro del área de la Didáctica de las Ciencias Sociales, en especial durante las dos últimas décadas. El uso del patrimonio con fines didácticos -en museística, archivística o arqueología- ha contribuido a la defensa de varias tesis doctorales (Miralles et al., 2011b, 161). Vinculada a ella, otra línea de investigación fructífera ha sido la que centra su atención en la forma 
en cómo los alumnos comprenden y le dan sentido a la historia, bien razonando a partir de textos o bien construyendo textos de historia. Asimismo, algunos investigadores del ámbito anglosajón se han pr eocupado en la denominada "formación de se ntido histórico" de los estudiantes o de la "construcción de I a co nciencia hi stórica". E s i nteresante co mprobar có mo v arios trabajos orientados a di cho obj etivo dest acan que al gunos estudiantes de $s$ ecundaria co nstruyen $\mathrm{s} u$ pensamiento histórico, a través de "competencias narrativas", desechando en par te otras fuentes de i nformación hast a e se $\mathrm{m}$ omento ar raigadas como l a familia, el ci ne y su s experiencias personales (Henríquez y Pagès, 2004, 68-70).

Los docentes de Ciencias Sociales debemos tener visiones diacrónicas amplias y contextualizar los hechos en tipos de formaciones sociales que, por su estructura económica, ideológica, cultural y política, expliquen estos hechos de manera totalmente diversa según el contexto. Por ello, no es conveniente el recurso a los acontecimientos episódicos y ef emérides históricas que pretenden justificar i deas o legitimar r ealidades políticas actuales (Prats, 2000 , 78-80). P ara co nocer los hechos históricos mediante una t écnica de i nvestigación, so lo di sponemos de fuentes (restos arqueológicos, docu mentos de archivo, obras de arte, etc.) que su ponen un t rabajo de anál isis, crítica y r elación q ue co nlleva un co nocimiento ci ertamente esp ecializado. D e hech o, muchos docentes prefieren impartir la historia a través de libros de texto que ofrezcan las informaciones cerradas o mediante explicaciones magistrales en las que la actividad de los alumnos es pasiva (Prats, 2000, 86).

El patrimonio es un recurso que transmite valores de respeto y preservación y va más allá del conocimiento científico, ya que conecta con lo vivencial y el sentido estético de una sociedad determinada, inmerso en protagonista de la revolución didáctica que no se queda anquilosada en las aulas (Hernández, 2003, 457 y 460-461; Estepa, 2004 y Hernández y Serrat, 2002). El trabajo con fuentes primarias facilita al al umno y a I a al umna conocer l as variables de I os procesos sociales, el concepto abstracto de multicausalidad y el relativismo del propio conocimiento histórico. Otro aspecto didáctico destacable es favorecer la capacidad empática con sociedades alejadas en el tiempo e intentar obtener respuestas sobre el funcionamiento interno de una sociedad, cu ya co mparación co $n$ est ructuras anteriores y post eriores of rece l a det ección de cambios y permanencias (Fernández y González, 2003, 556-557).

La ex plicación de I os pr ocesos sociales ha de $\mathrm{t}$ ener un se ntido crítico. Los trabajos de investigación previos en didáctica de la historia exponen el rendimiento óptimo en el proceso de aprendizaje a par tir del aprovechamiento di dáctico de I as fuentes primarias y la adq uisición de competencias básicas (Sáiz, 2013 y 2014): plantear problemas de aprendizaje histórico, analizar e interrogar a I as fuentes hi stóricas para co nstruir pr uebas del pasa do y desa rrollar r espuestas interpretativas redactando una narrativa histórica. La utilización de fuentes en el aula es una forma 
compleja de transmisión del conocimiento hi stórico, pero muy completa, al permitir obse rvar las argumentaciones, el planteamiento de relacionar procesos a través de causas y consecuencias y la comprensión de las transformaciones anejas a los hechos expuestos (Gómez et al., 2014). E trabajo con documentos proporciona el contacto con los denominados contenidos de segundo orden, de finidos por l a pose sión o desp liegue de di ferentes estrategias o c apacidades para responder a cu estiones hi stóricas y ent ender de una forma más compleja el pasa do ( Gómez, 2014, 134). Asimismo, el trabajo con fuentes permite al alumnado introducirse en el conocimiento de la historia, añade un carácter científico a la disciplina, que se muestra en continua construcción y revisión, y genera la adquisición de las competencias históricas (De la Montaña, 2019, 41-43).

Carretero y Limón (1995, 45-47) alertaban de la dificultad que tienen los alumnos y las alumnas para apreciar el conocimiento histórico y concebir al historiador "como un investigador de fuentes primarias y secundarias", ya que no distinguen lo que es evidencia -una información privilegiada sobre acontecimientos del pasado- de lo que es información. Estos autores recogen los distintos estadios descritos por Shemilt en 1987 sobre las ideas que posee el al umnado acerca de las fuentes históricas y el trabajo del historiador, donde se aprecia desde el desconocimiento inicial de los tipos de fuentes primarias hasta I a co ntextualización $\mathrm{f}$ inal de est as gracias a l as fuentes secundarias. La creación de un contexto histórico supone alcanzar la conciencia de la historicidad inherente a la evidencia.

El recurso a las fuentes primarias en la explicación didáctica de la historia ha quedado reflejado en diversos estudios como una vía al tamente recomendable para obtener un rendimiento óptimo en el aprendizaje significativo de los alumnos y las alumnas de las etapas de Educación Primaria y Secundaria ( Santisteban y $P$ agès, 2011). El a nálisis y el co mentario de $t$ extos y $f$ uentes documentales permiten adquirir $v$ arias de Ias competencias básicas recogidas en el cu rrículo actual, en especial la comunicación lingüística, la autonomía e iniciativa personal y la competencia social y ciudadana. El objetivo de la enseñanza de las Ciencias Sociales se ha de dirigir hacia el análisis de fuentes, la ejemplificación de los cambios y las permanencias, la interpretación de las causas y las consecuencias, incluso, el anál isis comprometido del se ntido ético de la disciplina histórica ( López et al., 2017). El uso de e stas fuentes primarias puede pr oporcionar una s herramientas de trabajo que conducen a la creación del pensamiento histórico en los alumnos y en las alumnas de I os distintos niveles educativos, como una muestra de metodología act iva (Gómez y Prieto, 2016; Gómez y Miralles, 2017; Gómez et al., 2018; Molina, 2011; Ortega, 2016 y 2019; Prieto et al., 2013).

Por úl timo, o tros trabajos vindican el uso de fuentes históricas para la identificación, el análisis temporal y la problematización de las representaciones hegemónicas, en especial para estudiantes de 14 a 18 años. Los alumnos yl as alumnas elaboran su propia reflexión 
argumentada a partir de las diferentes fuentes que se les han ido proporcionando, confrontando la representación narrativa del libro de texto con otros puntos de vista. Así los estudiantes se enfrentan a la evidencia de la pluralidad y la complejidad del conocimiento histórico, para analizar los diferentes relatos e interpretar de forma autónoma la cuestión hi stórica que se les presente (Rivière, 2020).

\subsection{La aportación didáctica de los archivos: el valor del patrimonio documental}

El conocimiento y la difusión del patrimonio histórico cumplen una función social que pone en valor y legitima los bienes culturales según los intereses de cada época. García y Jiménez (2003, 273274) comentan que «los archivos deben alcanzar el mismo rango que los museos en la enseñanza de las Ciencias Sociales», cuando apenas se contemplan como bien cultural, ya que su interés es minoritario y su acce so está r estringido pa ra primar la labor del investigador y la necesidad de conservación de los documentos. Los archivos pueden ser útiles en la construcción del conocimiento social, aunque en los contenidos curriculares las posibilidades educativas de los documentos como fuentes primarias quedan desdeñadas. Estas autoras señalan algunas claves y valores sobre el patrimonio documental:

- Es una rica manifestación del pat rimonio hi stórico-artístico. S u na turaleza puede se r escrita, gráfica, sonora, fílmica, videográfica o informática. En la última década se ha disparado el número de documentación digitalizada en los principales centros archivísticos.

- Posee la información su sceptible de co nvertirse en co nocimiento histórico a par tir de una investigación empírica.

- Adquiere una gran utilidad como recurso para aproximar al alumnado para acercar a los alumnos y a las alumnas al método de investigación histórica, además de ser un elemento educativo, formativo y de sensibilización para su protección.

¿Qué rentabilidad social se obtiene del patrimonio documental desde la disciplina de la Didáctica de las Ciencias Sociales? Aunque tradicionalmente ha existido un esca so desarrollo de políticas de di fusión documental, salvo excepciones generalmente de i nstituciones locales, la tarea de I a disciplina es divulgar su valor y contenido junto a la administración, diseñando infraestructuras y servicios a partir de criterios didácticos. El archivo es algo vivo y, por ello, debe estar abierto a la escuela co mo $r$ ecogen I as leyes protectoras del pat rimonio hi stórico. E n la úl tima déca da, aproximadamente, ha proliferado la utilización de los archivos como centros de interpretación que cuentan co $\mathrm{n}$ eq uipamiento y se rvicios adecuados para of recer di ferentes talleres de hi storia adecuados a los niveles de los discentes. Es una simbiosis altamente positiva entre la escuela y el propio archivo (Serrat, 2002). 
Gutiérrez (2004) defiende la necesidad de integrar el patrimonio como un elemento del entorno, al proyectar el pasado en el presente partiendo de fuentes documentales de carácter muy diverso e introducir el método del historiador de forma inducida, realista y por descubrimiento.

Para introducir al alumnado de Educación Secundaria en el proceso de investigación histórica la visita al ar chivo es un pr ocedimiento di dáctico út il. E s una forma d e desp egarse del pape I ideológico de la historia cuando a través de ella se enseñan los valores democráticos del Estado. Puede ayudar a superar el desinterés adolescente por la materia histórica, cuyo contenido se memoriza pero no se comprende. La historia debe alejarse de la metodología de otras Ciencias Sociales, con objetivos distintos, y basar su enseñanza y aprendizaje en el análisis de su materia prima, las fuentes. El pasado requiere una formulación de hipótesis y una clasificación correcta de la información, además de una instrucción sobre la crítica interna y externa de la documentación.

Ubieto (1989) y Santacana (2002) abogan por la apertura de los archivos municipales desde los que realizar propuestas en forma de ficha para enseñar historia. El acceso directo a documentación de I a l ocalidad donde se ubi can l os centros escolares es un acicate par a la realización y l a m otivación di dáctica de est as act ividades. Las posibilidades de anál isis son enormes dentro de su y acimiento docu mental: I os libros de $r$ egistro ( bautismos, bodas y defunciones), I os libros de act as, I os testamentos, I a esc uela en el pasa do, I as quintas o reemplazos del ej ército, I os tributos y la co ntabilidad, I a sa nidad local, I a pr ensa per iódica, I a información gráfica, I a correspondencia pr ivada, et c. D e toda es ta d ocumentación se puede $\mathrm{n}$ obtener aspectos demográficos, económicos o sociológicos. La información puede procesarse a través de tablas, casillas y etiquetas, donde señalar los conceptos previos, los procedimientos utilizados, el t ipo de ar chivo, I a nat uraleza de I a i nformación y I as hipótesis que pueden formularse.

\subsection{Breve estado de la cuestión sobre Didáctica de las Ciencias Sociales y archivos}

La función social y cultural de los archivos, al albergar en su documentación la memoria colectiva de generaciones de pe rsonas de épo cas lejanas en el tiempo, es tá fuera de toda duda. Las realidades cotidianas individuales y I os comportamientos colectivos de una I ocalidad o una sociedad quedan reflejados en la documentación que custodian como organismos administrativos, producto de las actividades emanadas por las instituciones civiles, eclesiásticas o militares y las personas. Los archivos recogen los testimonios de los derechos y obligaciones de los ciudadanos de un lugar y un tiempo concretos y tienen una labor esencial en la salvaguarda del patrimonio documental, co mo centros culturales con proyección de se rvicio a I a s ociedad (Lozano et al ., 1996, 9).

En pa íses como Fr ancia, G ran B retaña o A lemania hay propuestas estatales coherentes y organizadas con di rectrices homogéneas respecto a I as visitas a archivos y a I a publicación de 
compilaciones documentales y propuestas di dácticas. E n est e se ntido, en E spaña hay ca sos aislados pero de gran calidad en la administración local y autonómica. $Y$ además se compite con el pat rimonio hi stórico-artístico de monumentos, museos o y acimientos arqueológicos (Vela, 2002).

La realización de talleres y propuestas didácticas a partir de la documentación custodiada en los archivos es una vía adecuada para acercar los archivos a un púb lico no especializado, como los investigadores, y contribuyen a su conocimiento y difusión en el ámbito escolar, como recurso y herramienta de aprendizaje de las Ciencias Sociales.

Hay aut ores que han e nfatizado I a validez di dáctica del trabajo co $n$ fuentes extraídas de l os archivos (Fernández y González, 2003, 555-556). El uso de los archivos sirve para potenciar la presencia de las fuentes primarias como engranaje del proceso de enseñanza y aprendizaje de conceptos y procedimientos históricos recogidos en el currículo de los distintos niveles educativos. Los alumnos podrán familiarizarse con un tipo de fuentes documentales en las que inspeccionar aspectos de la vida cotidiana de las sociedades históricas y a instituciones desconocidas o ignoradas. El profesorado ha de asu mir que la accesibilidad del archivo histórico es positiva para la educación primaria y secundaria, y no solo lugar de estudio del investigador especialista.

Las visitas a los archivos deben pautarse y prepararse con antelación. Una propuesta didáctica tipo podría incluir los siguientes puntos:

- Visita inicial del grupo al archivo, donde se conocen sus características y funciones: qué fondos alberga. El docente ha debi do seleccionar previamente documentos interesantes para el rango de edad acorde.

- Transcripción de I os documentos y búsq ueda de anacr onismos reconocibles, co mo muestra de la noción de cambio temporal a partir del lenguaje escrito.

- Utilización de fuentes primarias para facilitar el aprendizaje de conceptos tales como las variables sociales, I a m ulticausalidad y e I r elativismo del co nocimiento hi stórico, a sí co mo I a empatía histórica con sociedades alejadas en el tiempo.

Las fuentes archivísticas nos muestran pr otagonistas, formas de $v$ ida co tidiana, co nflictos sociales, instituciones, creencias, variables educativas, et c., un fondo de $r$ ecursos en l os que descubrir pervivencias o transformaciones del pasado. Esta comparación con el presente posibilita la detección de cambios y permanencias y dota a los alumnos de las herramientas para construir una narración histórica al desarrollar procedimientos de formulación y comprobación de hipótesis, y co tejarlas con las conclusiones ofrecidas porl as corrientes historiográficas (Fernández y González, 2003, 556-557). La fructífera relación entre la historiografía y la didáctica de la Historia 
ha q uedado r ecogida de forma fehaciente por al gunos t rabajos monográficos (Miralles et al ., 2011a).

Los alumnos pueden iniciarse en los procedimientos y conceptos básicos de la historia. Otras funciones que cumplen los documentos son la toma de conciencia del pasado como transmisor de conocimientos, así co mo l a pr eocupación por co nservar el pat rimonio de I as colectividades humanas. Por último, el alumnado puede alcanzar un sentido crítico al dudar de la veracidad de lo que está recogido en el escrito (Fernández y González, 2003, 558).

Gemma Tribó, una de I as investigadoras que más ha tratado la cuestión que nos atañe, afirma que ha de e nseñarse a i nvestigar co mo es trategia bási ca de aprendizaje y $r$ ealiza al gunos interrogantes: ¿Qué fuentes históricas son las más adecuadas para su uso didáctico? ¿Se pueden usar fuentes en todas las etapas educativas? ¿Cómo introducirlas? ¿Cuándo? ¿Cuántas? En el contexto de di versidad étnica y la construcción de nue vas identidades en $E$ uropa, as í co mo la gestación de nuev as relaciones sociales, las estrategias de ap rendizaje de hi storia con fuentes primarias de los archivos próximos al centro educativo presentan unas características que los hacen idóneos para mostrar el contraste de una so ciedad tradicional frente a ot ra moderna, y de explicitar el valor de lo local frente a lo global (Tribó, 2002, 48-49).

La socialización crítica y responsable de los ciudadanos no es solo tarea de la escuela o la familia, sino que hay nuevos escenarios que cumplen esa función, como los medios de comunicación, la educación no formal, la administración local o las instituciones culturales. Los archivos locales y provinciales so $n$ uno de los ámbitos institucionales y culturales que se identifican $\mathrm{m}$ ás con la comunidad viva. A las funciones de investigación y administración tradicionales, en Europa se añade la didáctica escolar dentro de la divulgación cultural hacia la población general (Tribó, 2001, 160).

Aunque los docentes no han i nvestigado en a rchivos salvo al guna sa lvedad ni los archiveros conocen los contenidos del curriculum educativo, ambos profesionales convergen en la selección de unos criterios didácticos de selección tras una reflexión previa condicionada por unos factores psicopedagógicos y de i ntervención en el aul a. Los fondos más adecuados para se leccionar fuentes didácticas son los archivos locales y comarcales porque di sponen de un el emento emocional adi cional al $\mathrm{t}$ ratar a suntos del pasa do de $\mathrm{l}$ a I ocalidad y so $\mathrm{n}$ cercanos al ce ntro educativo. El conocimiento de las características de una sociedad histórica a través de las fuentes nos aproxima al co nocimiento de i dentidades alejadas espacial y temporalmente, gracias a la empatía desarrollada por el alumnado. De ahí que en Primaria es útil trabajar con documentos que traten t emas de $v$ ida co tidiana ( oficios, sa nidad, esco larización) y, si es factible, que tengan ilustraciones (Tribó, 2002, 50-51). 
Desde la sociología constructiva se enuncian dos razones para trabajar con fuentes primarias: la escuela ha de pr omover el conocimiento de I a sociedad donde se vive como ci udadanos para comprender su funcionamiento y todo act o educativo se realiza en un co ntexto so cial co ncreto. Entre sus objetivos se recogen motivar al alumno a partir de los elementos socioafectivos del entorno, al incorporar al proceso de en señanza de la historia elementos de identificación positiva en relación al espacio y al tiempo estudiados. En zonas con grandes transformaciones sociales intensas y rápidas (migraciones, cambios técnicos, crecimiento urbanístico) las visitas a archivos locales y l a r ealización de peq ueñas investigaciones con fuentes primarias ayudan a cr ear vínculos de identificación y pertinencia. Además, se logra reconstruir la memoria colectiva truncada. La desaparición de modos de vida tradicionales y el influjo de las tecnologías provocan una gran desorientación en la captación del tiempo social. Las fuentes históricas sobre problemáticas actuales del entorno aportan una carga socioafectiva que facilita la motivación y su implicación en el proceso de apr endizaje. Se pretende lograr la búsqueda de so luciones a los problemas históricos planteados en las fuentes primarias (Tribó, 2001, 161-163).

Las variables pedagógicas del trabajo co $\mathrm{n} f$ uentes son di versas: i doneidad, se lección, secuenciación $\mathrm{i}$ nstructiva, $\mathrm{t}$ rasposición di dáctica, $\mathrm{m}$ odelo peda gógico, y ca pacidad d e transferencia cognitiva y otras como el nivel de aprendizaje, adecuación al currículo escolar... Según el grado de complejidad de la fuente, la intervención pedagógica será distinta (un trabajo de investigación o si mulación hi stórica), pero se busca rá un p otencial motivador. Se o rdena la fuente en l a programación del profesor y en la intervención en el aula. Y se ha de adecu ar los temas del cu rrículo y las capacidades y co nocimientos previos del al umno a l a act ividad procedimental propuesta y no so brevalorar estos métodos desligados de los conceptos para no caer en un "activismo didáctico absurdo". El lenguaje narrativo y descriptivo de la fuente es otro aspecto para tener en cuenta (Tribó, 2001, 169-171 y 2002, 54-55).

\subsection{Principales actividades y conceptos didácticos que ofrece el archivo}

Los principales conceptos que se pueden trabajar con los documentos de I os archivos son las sociedades históricas y l os cambios en el tiempo. Las visitas y la realización de I os talleres didácticos necesitan una pr eparación previa en el aula y un t rabajo posterior con el objetivo de evaluar la actividad llevada a cabo.

Se debe trabajar el tiempo histórico más cercano al alumno siempre que sea posible -un punto de partida con el que poder comparar los procesos y fenómenos sociales- para poder comprender el modus vivendi de sociedades muy alejadas a la nuestra. El conocimiento certero de las medidas del t iempo y I as unidades habituales de per iodización $\mathrm{h}$ istórica ( edades, ca lendarios, si glos, décadas, años) es una herramienta ne cesaria para ace rcarse a I as fuentes documentales, que muestran una dat ación cronológica. El tiempo hi stórico queda plasmado en la transformación o 
evolución de di stintos aspectos materiales, vivenciales o $\mathrm{m}$ entales comol as viviendas y su s enseres, el vestido, l as costumbres, el trabajo, la religiosidad o I as festividades (Lozano et al., 1996, 15). Algunos autores han plasmado el diseño de actividades sobre el trabajo con fuentes que abordan la sucesión temporal o la empatía histórica con un gr an aprovechamiento didáctico, dirigido al ap rendizaje de co mpetencias y el pensamiento cr ítico de al umnos de E ducación Secundaria (Sandoya, 2016, 130, 168, 192).

Algunos centros archivísticos han i do r ealizando, desd e hace dos déca das, dosi eres con contenidos didácticos basados esencialmente en I a explotación pr ocedimental de I as fuentes documentales. Entre otras propuestas, mostramos un ejemplo realizado para el ciclo de Secundaria (Lozano et al., 1996, 16-17):

- Utilización de diversas informaciones sobre historia personal: partida de bautismo, cartilla de la Seguridad Social, Libro de Familia, etc.

- Análisis de la escritura de diferentes fuentes primarias, desde el siglo XV al XX.

- Comparación de diferentes formas de vida, usos y costumbres a través de un texto de la Edad Moderna y otro actual.

- Realización de un ar chivo pr opio per sonal co $n$ docu mentos escolares, f amiliares, de amigos, etc., o realización de la historia de la familia.

- Realización de números y letras característicos de la época analizada.

- Comparación entre fuentes históricas e historiográficas.

- Realización de peq ueñas investigaciones con documentos seleccionados del archivo, de ámbito local.

- Posible valoración del edificio del archivo como introducción al arte y su comparación con otros edificios de otras épocas.

Para los alumnos y para las alumnas la visita didáctica al archivo supone un a fianzamiento de la valoración por el $r$ espeto y co nservación del pat rimonio hi stórico-artístico, y la pr otección del conocimiento documental de las fuentes históricas. Las propuestas para el aprovechamiento didáctico del archivo son variadas y han de adaptarse al nivel educativo al que van dirigidas. Entre otras actividades didácticas, se pueden su gerir: uni dades didácticas para l a co mprensión del archivo y el documento histórico o sobre un tema de Ciencias Sociales al partir de un documento, itinerarios de visita al edificio del archivo y sus estancias; guías que explican las características y función del ar chivo, carpetas con fotocopias, facsímiles, instrumentos de escritura, planos, etc.; talleres de escritura; juegos de simulación; exposiciones, etc. (Sebastiá y Blanes, 2000, 237). 
Una posible propuesta didáctica podría partir de una encuesta inicial a los alumnos sobre qué es un ar chivo hi stórico, q ué t ipología docu mental pi ensan q ue al bergan y por qué puede se $r$ de utilidad su consulta. También es interesante explicar cómo se busca y localiza un documento en el archivo. A co ntinuación, se r ealizan las definiciones de docu mento y archivo, $\mathrm{P}$ or úl timo, se elabora un ejemplo de ficha de observación para su análisis: el soporte, la conservación (grado de deterioro), el tipo de letra, el idioma, las expresiones desconocidas, la datación, la localización, la firma o rúbrica, los protagonistas y la temática (Sebastiá y Blanes, 1999).

Otra propuesta trabaja las fuentes primarias del Archivo P rovincial de Salamanca y la em patía histórica, indagando a través de los documentos testamentarios de los universitarios, cómo era la vida estudiantil en el siglo XVI (Rubio-Muñoz, 2019). Con el análisis de esta tipología documental afloran asp ectos sociales, eco nómicos y culturales bastante desconocidos sobre los modos de vida. La m etodología de apr endizaje por descubrimiento o indagación permite el acercamiento al método ci entífico del hi storiador. E n l a m isma l ínea se encu entra ot ra i nvestigación so bre la utilización de los libros de apeo o repartimiento, en el contexto del levantamiento morisco de 1568 en el espacio almeriense, para su análisis didáctico (Martínez y Ponce, 2003).

El itinerario didáctico del archivo se elabora y planifica por los profesionales que trabajan en él, cuya función es clasificar, preservar y mostrar la documentación conservada. Conocer la historia del archivo y del monumento que lo al berga, a menudo considerado patrimonio histórico, es un preámbulo i deal par a e xplicar su s funciones, su or ganización y l a t ipología docu mental que custodia. El archivo ha de se leccionar d docu mentos que recojan temáticas interesantes, que les inciten a i nvestigar y despertar su curiosidad; el análisis de estos documentos debe per mitirles profundizar en grandes hech os históricos; I as fuentes elegidas deben se $r$ de fácil l ectura y comprensión. Los temas pueden ser presentados como un enigma, enunciando una hipótesis sin resolver que los alumnos tendrán que investigar a través de la documentación seleccionada.

Las actividades didácticas ofrecidas por algunos archivos pueden estar relacionadas directamente con el aprendizaje basa do en j uegos o los retos de una i nvestigación formulando una hi pótesis (Gómez et al., 2018).

\section{Metodología y resultados: el uso de las fuentes documentales en talleres didácticos de archivos históricos provinciales}

La Junta de Comunidades de Castilla-La Mancha ha impulsado el desarrollo de visitas didácticas de estudiantes de Educación Primaria y Secundaria a I os archivos históricos provinciales de la región en I a úl tima déca da. Los talleres suponen una est rategia m etodológica de i ncalculable potencial didáctico por el uso y la manipulación de fuentes primarias y secundarias. Así, el Archivo 
Provincial de Toledo ha desarrollado una serie de talleres escolares destinados a mostrar algunas pautas de la escritura antigua, cómo era la vida cotidiana en la época de personajes relevantes como Cervantes o El Greco, o un pueblo toledano hace 250 años o un documento de Alfonso VIII (Flores, 2018).

El Ar chivo P rovincial de G uadalajara el aboró u n dossi er di dáctico en focado al primer ci clo de Educación Secundaria con un el enco de num erosos documentos del archivo y de ot ros, como el Municipal de Guadalajara, con los que trabajar de forma procedimental. La guía didáctica diseña una se rie de a ctividades y paut as de ex plotación de I as fuentes, pl asmadas sobre temáticas variadas: el servicio militar, el matrimonio, los impuestos hacendísticos y los censos, el abastecimiento, la vivienda y los oficios (Lozano et al., 1996, 23-39).

Por su parte, el Archivo Histórico Provincial de A lbacete, en la misma línea de i mplementación didáctica, destina talleres para Educación Primaria para iniciar a los alumnos en las tareas y las funciones del escribano y del archivero, o en Educación Secundaria, añade el aprendizaje de investigador, I a escr itura co $\mathrm{n}$ pluma o I a co nfección de un ár bol g enealógico (www.castillalamancha.es).

\subsection{Los talleres del Archivo Histórico Provincial de Cuenca y su uso didáctico}

Vamos a analizar en detalle los talleres didácticos llevados a cabo hasta el curso 2017-2018 por el Archivo Histórico Provincial de Cuenca. Algunos autores han trabajado con documentos extraídos de este archivo, como Fernández y González (2003, 558-566), que proponen varios modelos de trabajo, en el primero de el los a través de un protocolo notarial de 1824 se puede $r$ ealizar un análisis histórico est ructural cl ásico y t rabajar la dur ación y la cr onología, as í co mo m últiples conceptos institucionales, políticos, económicos y sociales. La causalidad de las transformaciones político-jurídicas de carácter liberal o la reacción absolutista y la conflictividad social existente.

En ot ros documentos exploran cu estiones relativas a la vi da co tidiana y la cu ltura material, a través del trabajo infantil y femenino en manufacturas textiles, la marginación social de los pobres, la alimentación y el vestido y una acu sación de hech icería a C atalina Morena, una m orisca del Castillo de Garcimuñoz, en un documento fechado en 1555.

El desarrollo metodológico de la visita al Archivo necesita una preparación previa en el aula, así como un trabajo posterior en la misma que culminará con la evaluación que determine el grado de aprendizaje di dáctico de la experiencia. Los talleres están de stinados a al umnos de Educación Infantil, Primaria, Secundaria y Bachillerato de centros de la capital conquense y su provincia. En ellos, además de mostrarles las instalaciones y actividades desarrolladas en el Archivo, se les da a conocer el valor del patrimonio documental como fuentes primarias para el estudio de la Historia. Entre los objetivos específicos de los talleres se pueden enumerar los siguientes: 
- Conocer, diferenciar y utilizar las distintas tipologías de fuentes primarias e historiográficas que posee la institución.

- Desarrollar procedimientos de búsqueda de información que emulan la metodología de la investigación histórica.

- Estimular la curiosidad y el conocimiento por los hechos del pasa do y sus relaciones de causalidad histórica.

- Señalar la importancia de la conservación y la difusión del pat rimonio documental co mo legado a las futuras generaciones.

Los títulos de los talleres del Archivo Histórico Provincial de C uenca que se han desa rrollado en los últimos años son:

- "El Archivo", para los ciclos de Educación Infantil y de $1 .^{\circ}$ y $2 .^{\circ}$ de Educación Primaria. A través de unos dibujos y fotografías, los alumnos aprenden algunos conceptos para ser un archivero: planero, pergamino, cajas o secante. Por último, elaboran un sencillo árbol genealógico adaptado a su edad. El alumnado que realiza la visita conoce el mobiliario para el almacenamiento de los documentos y las posibilidades de reproducción y digitalización de los mismos.

- "Aprendiz de Escribano", destinado a $5 .^{\circ}$ y $6 .^{\circ}$ de Primaria. Se explica a los alumnos y a las alumnas cómo trabajan los escribanos, los utensilios que utilizaban para la escritura, el tipo de letra que utilizaban, cómo eran los soportes documentales (pergamino y papel) y las firmas que plasmaban al final de los documentos para dar fe del contenido recogido. También escriben sus nombres con un tipo de letra medieval decorada.

- "La Ciudad Medieval", destinado a al umnos del se gundo y tercer ci clo de E ducación Primaria y del primer ciclo de Educación Secundaria. Los discentes manejan un plano de la ciudad de Cuenca en época medieval, elaborado por Julio González, para señalar sus elementos claves. En esta fuente secundaria se aprecia la muralla que conformaba el perímetro fortificado de la ciudad, con sus distintas puertas de acce so. L os principales edificios que se plasman so $n$ el castillo, la catedral y las doce parroquias de las collaciones (barrios) conquenses. Extramuros se aprecia el hospital de Santiago, el molino de Santiago en el río Júcar y la albufera contigua a la desembocadura del río Huécar. Son elementos que ofrecen datos de interpretación económica y social, que completan las funciones militares, políticas y religiosas de la ciudad bajomedieval.

En se gundo I ugar, analizan un plano en una ca sa con su s estancias: patio, co cina, sala, horno y pajar. Al resolver un cr ucigrama despejan el concepto de escribano. Por último, rellenan un árbol genealógico más completo que el anterior, con el nombre de familiares que incluyen hasta los tatarabuelos. 
- "Interpretación de documentos sobre la Guerra de la Independencia y de las Guerras Carlistas", destinado a Bachillerato. Se analizan documentos custodiados en el archivo con datos geográficos, políticos e institucionales de ambos contextos bélicos en la provincia de Cuenca. La lectura de algunos conceptos históricos y personajes importantes, los datos sobre acciones concretas de índole político y militar, así como la cronología concreta explícita, permiten la elaboración de un contexto histórico aproximado de la época a la que pertenecen los documentos y la utilización de destrezas propias de la enseñanza del tiempo histórico, como la causalidad de los sucesos o los cambios o permanencias de determinadas estructuras políticas o sociales.

Los talleres, que tienen una dur ación aproximada de una hor a y media, finalizan con una visita guiada por las salas en que se ubicaban las antiguas celdas de la cárcel del Tribunal de la Inquisición, donde pued en ver y leer un so neto escrito a p rincipios del siglo XVII por uno de I os presos en la pared de su celda. Los alumnos han podido en su visita conocer y valorar la función de I as distintas ocupaciones profesionales relacionadas con I a cu stodia, I a co nservación y la difusión del patrimonio documental de los archivos, observando de primera mano legajos antiguos con docu mentos escritos en papel y per gamino. Conocer de pr imera $\mathrm{m}$ ano est as cuestiones garantiza que los escolares adquieran conciencia de la importancia de conservación y transmisión del legado documental, como muestra de la memoria colectiva de las épocas históricas pasadas.

\subsection{Evaluación de los talleres y sus actividades}

La ev aluación de I as actividades de los talleres puede ir enca minada a asi milar I os principales conceptos explicados en el los relacionados con I a di dáctica de I a historia, en I a co rrecta interpretación de I as funciones de los archivos y por qué es tan importante par a la so ciedad preservar y dar a conocer sus contenidos. La imitación de la caligrafía, los sellos de los escribanos o la elaboración de árboles genealógicos son estrategias didácticas de tipo receptivo y reactivo y favorecen la interactividad manual y la creatividad de I os alumnos y las alumnas. En los talleres destinados a cursos superiores se valora la interpretación co rrecta de los documentos (fuentes escritas, planos) y la creación de un contexto adecuado en el que cobra sentido la fuente primaria o secundaria analizada, como ejemplos de actividades de tipo reflexivo.

A través de una rúbrica evaluamos la actividad de cada uno de los talleres didácticos del archivo. Los resultados pueden or ientar al doce nte de I a co nsecución de I os o bjetivos de apr endizaje planteados con el manejo de las fuentes documentales, la materia prima para la construcción de conocimiento hi stórico. Se pueden adap tar I os elementos de $v$ aloración co nforme al ni vel educativo de los alumnos. En este caso exponemos una matriz de evaluación utilizando la escala de valoración tipo Likert. 


\begin{tabular}{|c|c|c|c|c|c|}
\hline Elementos de valoración & Deficiente & Mejorable & Bien & Muy bien & Excelente \\
\hline $\begin{array}{l}\text { Describe q ué es un } \\
\text { documento }\end{array}$ & & & & & \\
\hline $\begin{array}{l}\text { Identifica las funciones de } \\
\text { los archivos }\end{array}$ & & & & & \\
\hline $\begin{array}{l}\text { Redacta algunos } \\
\text { conceptos de los archivos } \\
\text { con buena caligrafía }\end{array}$ & & & & & \\
\hline $\begin{array}{l}\text { Dibuja el se llo de un } \\
\text { escribano siguiendo un } \\
\text { modelo }\end{array}$ & & & & & \\
\hline $\begin{array}{l}\text { Elabora un se ncillo ár bol } \\
\text { geneaológico }\end{array}$ & & & & & \\
\hline
\end{tabular}

Tabla. 1. Evaluación del taller didáctico "Aprendiz de escribano"

\begin{tabular}{|l|l|l|l|l|l|}
\hline Elementos de valoración & Deficiente & Mejorable & Bien & Muy bien & Excelente \\
\hline $\begin{array}{l}\text { Distingue entre f uentes } \\
\text { primarias secundarias }\end{array}$ & & & & & \\
\hline $\begin{array}{l}\text { Enumera las funciones de } \\
\text { los archivos }\end{array}$ & & & & \\
\hline $\begin{array}{l}\text { Obtiene i nformación } \\
\text { histórica de un pl ano: } \\
\text { partes esenciales y os } \\
\text { principales edificios de } \\
\text { una ciudad medieval }\end{array}$ & & & & & \\
\hline $\begin{array}{l}\text { Identifica características } \\
\text { sociales, eco nómicas y } \\
\text { culturales de una épo ca } \\
\text { histórica árbol }\end{array}$ & & & & & \\
\hline $\begin{array}{l}\text { Elabora un } \\
\text { geneaológico complejo }\end{array}$ & & & & \\
\hline
\end{tabular}

Tabla. 2. Evaluación del taller didáctico "La Ciudad Medieval"

\begin{tabular}{|l|l|l|l|l|l|}
\hline \multicolumn{1}{|c|}{ Elementos de valoración } & Deficiente & Mejorable & Bien & Muy bien & Excelente \\
\hline $\begin{array}{l}\text { Distingue entre f uentes } \\
\text { primarias y secundarias }\end{array}$ & & & & & \\
\hline $\begin{array}{l}\text { Clasifica I as fuentes } \\
\text { documentales }\end{array}$ & & & & & \\
\hline $\begin{array}{l}\text { Enumera las funciones de } \\
\text { los archivos eguntas }\end{array}$ & & & & & \\
\hline $\begin{array}{l}\text { Realiza pr os oncillas } \\
\text { sen obt ener } \\
\text { documentos para obt } \\
\text { información histórica }\end{array}$ & & & & \\
\hline $\begin{array}{l}\text { Identifica ca racterísticas } \\
\text { políticas, sociales, y eco- } \\
\text { nómicas de una época }\end{array}$ & & & & & \\
\hline
\end{tabular}

Tabla. 3. Evaluación de del taller didáctico "El Archivo" 


\begin{tabular}{|c|c|c|c|c|c|}
\hline Elementos de valoración & Deficiente & Mejorable & Bien & Muy bien & Excelente \\
\hline $\begin{array}{l}\text { Distingue entre f uentes } \\
\text { primarias y secundarias }\end{array}$ & & & & & \\
\hline $\begin{array}{l}\text { Clasifica la s fuentes } \\
\text { documentales y su } \\
\text { contenido temático }\end{array}$ & & & & & \\
\hline $\begin{array}{l}\text { Extrae I a i dea pr incipal y } \\
\text { las secundarias del } \\
\text { documento }\end{array}$ & & & & & \\
\hline $\begin{array}{l}\text { Realiza pr eguntas } \\
\text { complejas a l os } \\
\text { documentos para elaborar } \\
\text { un contexto histórico }\end{array}$ & & & & & \\
\hline $\begin{array}{l}\text { Identifica ca racterísticas } \\
\text { políticas, m ilitares y } \\
\text { sociales de una épo ca } \\
\text { histórica }\end{array}$ & & & & & \\
\hline
\end{tabular}

Tabla. 4. Evaluación de del taller didáctico "Interpretación de documentos"

\section{Conclusiones}

El uso de I as fuentes primarias, en primer lugar, conduce a l a creación de pensa miento histórico en los alumnos. En segundo término, el pensamiento histórico crítico se profundiza en contenidos conceptuales y a t ravés de I a asi milación de pr ocedimientos. P or úl timo, I a i nclusión d e documentos primarios en el proceso de aprendizaje de la historia ayuda a la adquisición de las competencias básicas, cu ya interiorización s upone una ut ilización habitual de co ntenidos procedimentales a través de la variedad de recursos y materiales que permiten su evaluación posterior (Miralles et al., 2012). Es cierto, no ob stante, que el anál isis de fuentes requiere una "lectura di sciplinar" pr evia par a poder "escribir hi stóricamente", I abor d ocente m ás compleja al incluir vínculos intertextuales y explicaciones relacionales (Sáiz, 2014, 88).

A par tir del anál isis de fuentes documentales conservadas en I os archivos, co mo he mos observado, se pueden abor dar al gunos de l as dificultades que co ncierne l a ense ñanza de la Historia, co mo r ecogía el profesor Prats (2000, 87 -97): en primer lugar, el apr endizaje de conceptos históricos, términos que tienen una utilización co rriente en el lenguaje cotidiano, por ejemplo: monarca, absolutismo, protestantismo, etc., de significación diversa y, en ocasiones, muy distinta según el contexto histórico en el que se trate para construir conocimiento histórico, pero cuya complejidad es notoria al delimitar su carga descriptiva e interpretativa. En segundo lugar, la percepción del t iempo en hi storia, cu ya co mplicación co nlleva l a d istinción ent re l os diversos ritmos de cambio. Ya sabemos que la concepción temporal viene condicionada por la consecución del pensamiento formal y que su aprendizaje tiene un carácter constructivo. 
El trabajo de los alumnos con fuentes y la visita al archivo puede encuadrarse en una metodología marco co mol a $r$ esolución de si tuaciones-problema en pe queños grupos de trabajo. Los estudiantes crean un pr oceso de i ndagación que resuelve preguntas y dudas sobre fenómenos complejos, y tratan de comprender el pr oblema y de busca $r$ una es trategia de so lución que conlleva el razonamiento científico, al plantear una hipótesis. En última instancia, se debate y se critican procesos históricos aparentemente cerrados, por lo que queda constatada la licitud de creación, análisis e interpretación de nuevos contenidos históricos (Gómez et al., 2018).

\section{Agradecimientos}

Este t rabajo es resultado del pr oyecto de i nvestigación "Conceptos metodológicos y m étodos activos de aprendizaje para la mejora de las competencias docentes del profesorado" (PGC2018094491-B-C33) (MCI/AEI/FEDER, UE), subvencionado por el Ministerio de C iencia, Innovación y Universidades y cofinanciado con fondos FEDER de la UE.

\section{Referencias}

Carretero, M. y Limón, M. (1995). Construcción del conocimiento y enseñanza de las Ciencias Sociales y la Historia. En M. Carretero (coord). Construir y enseñar. Las Ciencias Sociales y la Historia. Visor.

De la Montaña Conchiña, J. L. (2019). Las fuentes documentales como recursos para la enseñanza de la Historia. En A. M. Hernández C arretero (coord.). Estrategias y recursos para la enseñanza de las Ciencias Sociales. (pp. 37-53). Ediciones Pirámide.

Estepa Giménez, J. (2004). El patrimonio documental y los archivos como recursos en la enseñanza de las Ciencias S ociales. En R. R ey de I as P eñas (coord.). Aprender y enseñar con el archivo: séptimas jornadas archivísticas. Diputación Provincial de Huelva. 33-46.

Fernández Valencia, A. y González Marzo, F. (2003). Uso didáctico del legado histórico conservado en los archivos. En E. Ballesteros Arranz, C. Fernández Ruiz, J. A. Molina Ruiz y P. Moreno Benito (coords.). El patrimonio y la didáctica de las Ciencias Sociales. (pp. 555-568) Universidad de CastillaLa Mancha.

Flores Varela, C. (2018). La difusión desde los Archivos Históricos Provinciales: el caso de Toledo. Boletín de la $A N A B A D, 68(3-4), 462-482$

García R uiz, C . R . y J iménez Martínez, M. D . (2003). E I p atrimonio doc umental en I a di dáctica de I as Ciencias Sociales. En E. Ballesteros Arranz, C. Fernández Ruiz, J. A. Molina Ruiz y Moreno Benito P. (coords.). El patrimonio y la didáctica de las Ciencias Sociales. (pp. 271-280). Universidad de CastillaLa Mancha. 
Gómez Carrasco, C. J. (2014). Pensamiento histórico y contenidos disciplinares en los libros de texto. Un análisis exploratorio de la Edad Moderna en $2{ }^{\circ}$ de la ESO. ENSAYOS. Revista de la Facultad de Educación de Albacete, 29(1), 131-158.

Gómez Carrasco, C. J. y Prieto Prieto, J. A. (2016). Fuentes primarias, objetos y artefactos en la interpretación de la $\mathrm{h}$ istoria. $\mathrm{D}$ iseño y evaluación de $u \mathrm{nt}$ aller $\mathrm{d}$ e num ismática en $\mathrm{E}$ ducación Secundaria. Didáctica de las Ciencias Experimentales y Sociales, 31(2), 5 -22. https://doi.org/10.7203/dces.31.8228

Gómez Carrasco, C . J .; O rtuño Molina, J. y M olina Puche, S . (2014). A prender a pe nsar hi stóricamente. Retos para la historia en el siglo XXI. Tempo e Argumento, 6(11). 5-27.

Gómez Carrasco, C. J. y Miralles Martínez, P. (2017). Los espejos de Clío. Usos y abusos de la Historia en el ámbito escolar. Sílex Ediciones.

Gómez C arrasco, C . J ., O rtuño Molina, J . y Miralles Martínez, P. (2018). Enseñar ciencias sociales con métodos activos de aprendizaje. Reflexiones y propuestas a través de la indagación. Octaedro.

Gutiérrez, M. L. (2004). El patrimonio y la historia: el análisis de fuentes históricas mediante un estudio de caso. Iber. Didáctica de las ciencias sociales, geografía e historia, 42, 109-126.

Henríquez, R. y Pagés i Blanch, J. (2004). La investigación en di dáctica de la historia. Educación XX1. Revista de la Facultad de Educación. UNED, 7, 63-84.

Hernández Cardona, F. X. (2003). El patrimonio como recurso en la enseñanza de las Ciencias Sociales. En E. Ballesteros Arranz, C. Fernández Ruiz, J. A. Molina Ruiz y P. Moreno Benito (coords.). El patrimonio y la didáctica de las Ciencias Sociales. (pp. 455-466). Universidad de Castilla-La Mancha.

Hernández Cardona, F. X. y Serrat Antolí, N. (2002). Los archivos en la didáctica de las Ciencias Sociales. Iber Didáctica de las ciencias sociales, geografía e historia, 34, 5-6.

López F acal, R ., Mi ralles Mar tínez, P ., y P rats C uevas ( dirs.) y G ómez C arrasco, C . J . (coord.) (2017). Enseñanza de la historia y competencias educativas. Graó.

Lozano Leal, F., Torres Ortez, H., Santana Núñez, M. ${ }^{a}$ I. y Mejía Asensio, Á. (1996). El Archivo, un centro vivo y abierto a la actividad escolar: propuesta didáctica. Junta de $\mathrm{C}$ omunidades de $\mathrm{C}$ astilla-La Mancha.

Martínez Lóp ez, J. M. y P once Mo lina, P. (2003). Las actividades en Ciencias Sociales. E I pat rimonio documental: I os I ibros de apeo/repartimiento c omo r ecurso di dáctico. En E. Ballesteros A rranz, C . Fernández Ruiz, J. A. Molina Ruiz y P. Moreno Benito (coords.). El patrimonio y la didáctica de las Ciencias Sociales. (pp. 475-488). Universidad de Castilla-La Mancha.

Miralles Martínez, P., Molina Puche, S. y Ortuño Molina, J. (2011a). La importancia de la historiografía en la enseñanza de la historia. Grupo Editorial Universitario.

Miralles M artínez, P., Mo lina Puche, S. y O rtuño Molina, J . (2011b). La investigación en D idáctica de la Ciencias Sociales. Educatio Siglo XXI, 29(1), 149-174. 
Molina Puche, S. (2011). El uso de la genealogía para la enseñanza de la Historia en los niveles educativos iniciales. Clío. HIstory and History teaching 37, 1-19.

Ortega Cervigón, J. I. (2016). Un acercamiento al aprendizaje significativo de la historia: la utilización de la cronística de C arlos V en el au la. En F. García González, C. J. Gómez Carrasco, R. A. Rodríguez Pérez (eds.). La Edad Moderna en la Educación Secundaria. Experiencias de investigación. (pp. 2738). Universidad de Murcia: Servicio de Publicaciones.

Ortega Cervigón, J. I. (2019). ¿Es viable un taller de Paleografía en Educación Primaria? La utilización de las fuentes históricas en el aula. Enseñanza de las Ciencias Sociales. Revista de Investigación, 18, 35-47. https://doi.org/10.1344/ECCSS2019.18.3

Prats Cuevas, J. (2000). Dificultades para la enseñanza de la Historia en la Educación Secundaria: reflexiones ante la situación española. Revista de Teoría y Didáctica de las Ciencias Sociales, 5, 7198.

Prieto Prieto, J. A., Gómez Carrasco, C. J. y Miralles Martínez, P. (2013). El uso de fuentes primarias en el aula y el desarrollo del pensamiento histórico y social. Una experiencia en Bachillerato. Clío. History and History teaching, 39.

Rivière Gómez, A. (2020). Repensar la enseñanza de la Historia. En Rivière Gómez, A. (coord.). Hacia una enseñanza de la Historia renovada. Reflexiones críticas y propuestas didácticas. ACCI. 9-58.

Rubio-Muñoz, F. J. (2019). La visibilidad de lo cotidiano. Didáctica, historia y fuentes documentales para el estudio d e la vida universitaria en I a S alamanca m oderna. El Futuro del Pasado, 10, 373-392. https://doi.org/10.14516/fdp.2019.010.001.014

Sáiz Serrano, J. (2013). Alfabetización histórica y competencias básicas en libros de texto de historia y en aprendizaje de estudiantes. Didáctica de la Ciencias Experimentales y Sociales, 27, 43-66. https://doi.org/10.7203/dces.27.2648

Sáiz Serrano, J. (2014). Fuentes históricas y libros de texto en Secundaria: una oportunidad perdida para enseñar competencias de pensamiento histórico. ENSAYOS, Revista de la Facultad de Educación de Albacete, 29(1), 83-99.

Sandoya Hernández, M. A. (2016). Enseñar Ciencias Sociales. 35 actividades para desarrollar capacidades. UOC.

Santisteban, A. y Pagès, J. (2011). Enseñar y aprender el tiempo histórico. En A. Santisteban y J. Pagès (coords.). Didáctica del Conocimiento del Medio Social y Cultural en la Educación Primaria. Ciencias Sociales para aprender, pensar y actuar. (pp. 229-247). Síntesis.

Santacana, J. (2002). La investigación en ar chivo: pautas y propuestas para la es cuela s ecundaria. Iber. Didáctica de las ciencias sociales, geografía e historia, 34, 7-20.

Sebastià i A Icaraz, R. y Blanes N adal, G. (1999). El aprovechamiento didáctico de los archivos y su concreción en el Archivo Municipal de Alcoi. Editorial Club Universitario. 
Sebastià i A lcaraz, R. y B lanes Nadal, G. (2000). El ar chivo y su di dáctica. En J. Pagés, J. Estepa y G. Trabé (eds.). Modelos, contenidos y experiencias en la formación del profesorado de Ciencias Sociales. (pp. 235-252). Universidad de Huelva.

Serrat Antolí, N. (2002). Una simbiosis archivo-escuela. Iber. Didáctica de las ciencias sociales, geografía e historia, 34, 27-36.

Tribó T raveria, G . (2001). A rchivos, fuentes y d idáctica de la Historia. Historia, Antropología y Fuentes Orales. ¿Confiar en la Historia?, 25, 159-176.

Tribó Traveria, G. (2002). Archivos municipales y comarcales y didáctica de la historia. Iber. Didáctica de las ciencias sociales, geografía e historia, 34, 46-57.

Ubieto Arteta, A. (1989). Archivos locales y didáctica de la h istoria: u tilización de fuentes de acceso fácil para el estudio de la localidad y del entorno. Educación Abierta, Aspectos didácticos de Geografía e Historia (Historia, 4), 74. 11-53.

Vela, S. (2002). A rchivos y didáctica: u n es tado de I a cuestión. Iber. Didáctica de las ciencias sociales, geografía e historia, 34, 21-26.

Publicado bajo licencia internacional Creative Commons Attribution-NonCommercial-ShareAlikeNoDerivs 4.0 (CC-BY-NC-SA 4.0). Se permite copiar, us ar, di stribuir, transmitir y exhibir públicamente, siempre que: i)se reconozca la autoría y la fuente or iginal de publ icación(revista, e ditorial y U RL de I t rabajo); ii) no s e ut ilice con fines comerciales; iii) se mencione la existencia y especificaciones de esta licencia. 